\title{
The Sharpe ratio of estimated efficient portfolios
}

\author{
Apostolos Kourtis*
}

First version: June 62014

This version: January 232016

\begin{abstract}
Investors often adopt mean-variance efficient portfolios for achieving superior risk-adjusted returns. However, such portfolios are sensitive to estimation errors, which affect portfolio performance. To understand the impact of estimation errors, I develop simple and intuitive formulas of the squared Sharpe ratio that investors should expect from estimated efficient portfolios. The new formulas show that the expected squared Sharpe ratio is a function of the length of the available data, the number of assets and the maximum attainable Sharpe ratio. My results enable the portfolio manager to assess the value of efficient portfolios as investment vehicles, given the investment environment.
\end{abstract}

Keywords: Portfolio performance; mean-variance analysis; estimation errors

JEL Classification: C13; G1

\footnotetext{
*Norwich Business School, University of East Anglia, Norwich, Norfolk, NR4 7TJ, UK, a.kourtis@uea.ac.uk. Tel. +44 (0)1603 591387. I am grateful to the editor, Brian Lucey, and an anonymous referee for useful suggestions and comments.
} 


\section{Introduction}

Almost five decades have passed since William F. Sharpe introduced the expected excess return per unit of risk as a measure of investment performance (Sharpe 1966, 1994). Despite the plethora of alternatives that have been proposed by academics and practitioners, the Sharpe ratio remains one of the most popular metrics used to rank portfolios and investment companies. For example, Amenc et al. (2003) find that the majority of distributors of hedge funds measure risk-adjusted returns using the Sharpe ratio.

The extensive use of the Sharpe ratio couples with the wide adoption of portfolio strategies prescribed by Modern Portfolio Theory (MPT), pioneered by Markowitz (1952). ${ }^{1}$ According to MPT, the portfolio that maximizes the Sharpe ratio lies on the mean-variance efficient frontier. This portfolio corresponds to the point where the Capital Market Line is tangent to the frontier and, as such, it is known as the tangency portfolio. To implement the tangency portfolio, investors need to estimate expected returns, variances and covariances for the assets in the portfolio. As estimated portfolios are prone to errors, the maximum Sharpe ratio promised by MPT is unattainable in practice (e.g., see Michaud, 1989; DeMiguel, et al. 2009).

What is the Sharpe ratio that we should expect from estimated efficient portfolios? To answer this question, previous studies use simulations or experiments in real data (e.g., DeMiguel et al., 2009; Tu and Zhou, 2011). However, the lack of closed-form results does not allow a clear picture of the determinants of the Sharpe ratio for estimated portfolios. I address this issue by deriving a new set of simple and intuitive analytical formulas. These represent accurate approximations of the expected squared Sharpe ratio (SSR, hereafter) of the estimated tangency portfolio, i.e., the average SSR that an investor would attain if she follows this portfolio. ${ }^{2}$ In essence, the expected SSR is a measure of the out-of-sample

\footnotetext{
${ }^{1}$ See Grinold and Kahn (1999) and Meucci (2005) for examples of how MPT is applied in the investment practice.

${ }^{2}$ I adopt the squared Sharpe ratio instead of the ratio in its raw form, as the expected value of the first can be expressed in a more intuitive form. Treynor and Black (1973) and Grinold (1989), among many others, also use the squared ratio as a performance measure.
} 
performance of the tangency portfolio.

The new formulas identify the drivers of the Sharpe ratio for estimated efficient portfolios and quantify the impact of estimation errors. It turns out that the expected SSR is a function of three parameters that determine the investment setting, i.e., (i) the number of assets in the portfolio, (ii) the length of the sample of historical data available for estimation and (iii) the maximum Sharpe ratio offered by the ex-ante tangency portfolio. In particular, the expected SSR increases with the sample length and decreases with the number of assets, all else equal. This is because the higher the length of the sample or the lower the number of assets, the more precise the estimates of the portfolio weights tend to be. Moreover, the effect of estimation errors deteriorates for larger values of the maximum possible Sharpe ratio. I finally show how the portfolio manager can estimate the expected SSR of the estimated optimal portfolio using historical data.

This paper is related to the influential work of Lo (2002) on the distribution of the Sharpe ratio. A key difference between the work of Lo and this article is that the the first focuses on the asymptotic distribution of the ex-post Sharpe ratio while the quantity of interest here is the ex-ante squared Sharpe ratio. This article also extends the study of Miller and Gehr (1978) who obtain the distribution of the Sharpe ratio, assuming that portfolio weights are fixed. I instead consider weights estimated from historical data, as is typical in practice. Finally, the methods in this article are motivated by the work of Kan and Zhou (2007) who study out-of-sample portfolio performance in terms of expected certainty-equivalent returns. This article differs in using the squared Sharpe ratio as a performance measure.

\section{Investment setting}

In this section, I describe the portfolio choice process for a mean-variance investor who aims to maximize the Sharpe ratio. I consider a market of $N$ risky assets and a risk-free asset. The returns on the risky assets at time $t$ are denoted with $R_{t}$ while $R_{t}^{f}$ is the return on the 
risk-free asset. Let then $r_{t}=R_{t}-R_{t}^{f}$ be the returns on the risky assets in excess of the risk-free rate. Following a common convention in the literature (e.g., see, Kan and Zhou, 2007 and $\mathrm{Tu}$ and Zhou, 2011), I assume that $r_{t}$ are independent and identically normally distributed with mean $\mu$ and covariance matrix $\Sigma$. At each time $t$, the investor's objective is to maximize the Sharpe ratio:

$$
\frac{w^{\prime} \mu}{\sqrt{w^{\prime} \Sigma w}}
$$

subject to the constraint:

$$
w^{\prime} 1_{N}=1
$$

where $w$ are the portfolio weights on the risky assets and $1_{N}$ is an $N$-vector of 1 's. $w^{\prime} \mu$ and $w^{\prime} \Sigma w$ are the portfolio mean excess return and variance, respectively. The constraint (2) ensures that the investor chooses a portfolio of risky assets only.

The optimal portfolio for the investor is the tangency portfolio

$$
w^{*}=\frac{\Sigma^{-1} \mu}{1_{N} \Sigma^{-1} \mu}
$$

provided that the denominator is different than zero. The tangency portfolio offers the maximum Sharpe ratio

$$
\theta=\sqrt{\mu^{\prime} \Sigma^{-1} \mu}
$$

If investment in the risk-free asset is allowed, the optimal risky asset weights are proportional to the tangency portfolio and achieve the same Sharpe ratio.

In practice, the maximum Sharpe ratio $\theta$ cannot be obtained, because the expected returns, the variances and the covariances are unknown. As a remedy, practitioners use estimates of the unknown parameters to compute the portfolio weights. The estimation is typically performed using historical data. Here, I assume that the investor uses the common 
Maximum Likelihood estimator of the means: ${ }^{3}$

$$
\hat{\mu}=\frac{1}{T} \sum_{j=t-T+1}^{t} r_{t},
$$

where $T$ is the number of observations in the dataset available to the investor. I also assume that the investor knows the true value of the covariance matrix $\Sigma$. While uncertainty about the covariance matrix could be accommodated, this would come at the cost of mathematical tractability. Nevertheless, the means are significantly more difficult to estimate than variances-covariances (see Chopra and Ziemba, 1993). For example, investors can increase the frequency in the returns in their sample and greatly improve the estimation of $\Sigma$, but not of $\mu$ (see Merton, 1980).

The estimated tangency portfolio weights are $\hat{w}=\frac{\Sigma^{-1} \hat{\mu}}{1_{N} \Sigma^{-1} \hat{\mu}}$. Then, the Sharpe ratio of the estimated tangency portfolio is

$$
\hat{\zeta}=\frac{\left(\hat{w}^{\prime} \mu\right)}{\sqrt{\hat{w}^{\prime} \Sigma \hat{w}}} .
$$

This will generally be lower than $\theta$, because of estimation errors in $\hat{w}$.

When there are more than one asset in the portfolio, the Sharpe ratio of the estimated portfolio above is a random variable, as a function of the returns in the available dataset. Studying the stochastic properties of $\hat{\zeta}$ can help to understand how errors in the estimated portfolio $\hat{w}$ affect the Sharpe ratio. In this context, the next section derives an intuitive expression of the expected value of the squared Sharpe ratio $\hat{\zeta}^{2}$.

\footnotetext{
${ }^{3}$ Alternative estimators for $\mu$ that are normally distributed, such as shrinkage estimators, could also be considered here.
} 


\section{The expected squared Sharpe ratio}

The expected squared Sharpe ratio (SSR) $E\left(\hat{\zeta}^{2}\right)$ is the average SSR that the investor would attain, if she adopts the estimated tangency portfolio. ${ }^{4}$ When the Sharpe ratio is used as a performance measure, the expected SSR represents the average out-of-sample performance of the tangency portfolio. The next proposition derives two simple formulas for the expected SSR. The mathematical proof is contained in the Appendix.

Proposition 1 The expected SSR, E( $\left.\hat{\zeta}^{2}\right)$, for the estimated tangency portfolio can be approximated as

$$
\begin{array}{r}
\bar{\zeta}_{1}^{2}:=\theta^{2}-\frac{(N-1) \theta^{2}}{N+T \theta^{2}} \text { (first-order approximation) } \\
\bar{\zeta}_{2}^{2}:=\theta^{2}-\frac{(N-1) \theta^{2}}{N+T \theta^{2}}-\frac{2(N-1) T \theta^{4}}{\left(N+T \theta^{2}\right)^{3}} \text { (second-order approximation) }
\end{array}
$$

I study the precision of the above approximations using two datasets of real asset returns. ${ }^{5}$ They respectively contain monthly returns for the period 07/1967-12/2014 on 10 industry portfolios (10Ind) and on 25 portfolios formed on size-and-book to market (25SBM). The datasets are popular in the recent portfolio choice literature (for instance, see Kan and Zhou, 2007; DeMiguel et al., 2009). To compute excess returns, I use the 30-day T-Bill rate as the risk-free asset. For each dataset, I set $\mu$ and $\Sigma$ equal to the respective sample moments computed using the whole dataset. Then, the maximum SSR $\left(\theta^{2}\right)$ is 0.0366 for the 10Ind set and 0.2037 for the 25SBM set. I consider in my analysis four different sample lengths $(T=60,120,240$ and 480$)$, corresponding to $5,10,20$ and 40 years of data available to the investor. For each sample length, I compute both approximations $\bar{\zeta}_{1}^{2}$ and $\bar{\zeta}_{2}^{2}$ in each dataset. To assess their accuracy, I compare them to the true value of the expected SSR $\left(E\left(\hat{\zeta}^{2}\right)\right)$ computed using simulation. Table 1 reports the results from this experiment.

\footnotetext{
${ }^{4}$ The expectation is taken under the true distribution of asset returns.

${ }^{5}$ All data is obtained from Kenneth French's website (http://mba.tuck.dartmouth.edu/pages/faculty/ ken.french/data_library.html).
} 


\section{Table 1: Accuracy of the approximations of the expected squared Sharpe ratio (SSR)}

\begin{tabular}{|c|c|c|c|c|c|c|c|c|}
\hline \multirow[b]{2}{*}{$N$} & \multirow[b]{2}{*}{$\theta^{2}$} & \multirow[b]{2}{*}{$T$} & \multicolumn{3}{|c|}{ Expected SSR } & \multicolumn{3}{|c|}{ Expected loss in the SSR } \\
\hline & & & $\bar{\zeta}_{1}^{2}$ & $\bar{\zeta}_{2}^{2}$ & $E\left(\hat{\zeta}^{2}\right)$ & $\bar{\rho}_{1}$ & $\bar{\rho}_{2}$ & $\rho$ \\
\hline \multirow{4}{*}{10} & \multirow{4}{*}{0.0366} & 60 & 0.0096 & 0.0088 & 0.0089 & 0.0270 & 0.0278 & 0.0277 \\
\hline & & 120 & 0.0137 & 0.0127 & 0.0127 & 0.0229 & 0.0239 & 0.0239 \\
\hline & & 240 & 0.0190 & 0.0182 & 0.0181 & 0.0176 & 0.0184 & 0.0185 \\
\hline & & 480 & 0.0246 & 0.0241 & 0.0240 & 0.0120 & 0.0125 & 0.0126 \\
\hline \multirow{4}{*}{25} & \multirow{4}{*}{0.2037} & 60 & 0.0724 & 0.0700 & 0.0701 & 0.1313 & 0.1337 & 0.1336 \\
\hline & & 120 & 0.1048 & 0.1029 & 0.1028 & 0.0989 & 0.1008 & 0.1009 \\
\hline & & 240 & 0.1375 & 0.1364 & 0.1363 & 0.0662 & 0.0673 & 0.0674 \\
\hline & & 480 & 0.1639 & 0.1634 & 0.1634 & 0.0398 & 0.0403 & 0.0403 \\
\hline
\end{tabular}

Note: This table presents the value of the two approximations $\left(\bar{\zeta}_{1}^{2}\right.$ and $\left.\bar{\zeta}_{2}^{2}\right)$ of the expected squared Sharpe ratio (SSR) of the tangency portfolio, derived in section 3, for different values of the number of assets $N$ and the sample length $T$. The underlying assumption is that asset returns follow a normal distribution with parameters calibrated in a set of 10 industry portfolios $(N=10)$ and a set of 25 portfolios formed on size and book-to-market $(N=25)$. The true value of the expected $\operatorname{SSR}\left(E\left(\hat{\zeta}^{2}\right)\right)$, computed using simulation, is also reported. Finally, the table presents the corresponding approximations of the loss in SSR of the tangency portfolio due to estimation errors $\left(\bar{\rho}_{1}\right.$ and $\left.\bar{\rho}_{2}\right)$ as well as, the true value of the loss $(\rho)$, computed via simulation.

I find that $\bar{\zeta}_{2}^{2}$ is a very accurate approximation of $E\left(\hat{\zeta}^{2}\right)$ in all cases considered. The error in the simpler approximation $\bar{\zeta}_{1}^{2}$ ranges from $2 \%$ to $8 \%$ in the 10 Ind dataset and from $0.3 \%$ to $3 \%$ in the $25 \mathrm{SBM}$ dataset. The error appears to decrease with the length of the sample. As such, $\bar{\zeta}_{2}^{2}$ should be used for relatively small samples, instead of $\bar{\zeta}_{1}^{2}$.

The formulas in Proposition 1 allow me to quantify the impact of estimation errors on the out-of-sample portfolio performance. To this end, I consider the difference in the expected SSR between the estimated portfolio $\hat{w}$ and the ex-ante portfolio $w^{*}$ :

$$
\rho=\theta^{2}-E\left(\hat{\zeta^{2}}\right)
$$


In essence, $\rho$ is the expected loss in the SSR due to estimation errors. Based on Proposition 1, this can be approximated as

$$
\begin{gathered}
\bar{\rho}_{1}:=\theta^{2}-\bar{\zeta}_{1}^{2}=\frac{(N-1) \theta^{2}}{N+T \theta^{2}} \\
\bar{\rho}_{2}:=\theta^{2}-\bar{\zeta}_{2}^{2}=\frac{(N-1) \theta^{2}}{N+T \theta^{2}}+\frac{2(N-1) T \theta^{4}}{\left(N+T \theta^{2}\right)^{3}}
\end{gathered}
$$

The accuracy of $\bar{\rho}_{1}$ and $\bar{\rho}_{2}$ can also be examined via Table 1 . By construction, the secondorder approximation is more precise. Nevertheless, $\bar{\rho}_{1}$ accounts for more than $95 \%$ of the loss in SSR in most cases.

\section{What drives the Sharpe ratio?}

This section aims to identify the drivers of the Sharpe ratio of estimated efficient portfolios. Proposition 1 reveals that the expected SSR is a function of the number of assets $N$, the sample length $T$ and the maximum Sharpe ratio $\theta$ of the ex-ante tangency portfolio. All else equal, the expected SSR:

1. increases with the sample length.

2. decreases with the number of assets.

3. increases with the maximum Sharpe ratio. ${ }^{6}$

The above become clear when one considers the percentage loss in the SSR, computed using the first-order approximation:

$$
\frac{\rho}{\theta^{2}} \approx \frac{N-1}{N+T \theta^{2}}
$$

The last equation illustrates the impact of estimation errors on the out-of-sample portfolio performance. The higher the sample length or the lower the number of assets, the higher the out-of-sample Sharpe ratio will be, because the less the estimation errors in $\hat{\mu}$ tend to be.

\footnotetext{
${ }^{6}$ These findings can be confirmed via differentiation.
} 
The positive relationship between the expected SSR and the sample length is also evident in Table 1. For example, in the 10Ind set, the expected SSR is 0.009 when the sample contains 60 months of historical returns while it doubles to 0.018 for a sample of 240 returns. Another interesting finding from the above equation is that the higher the maximum Sharpe ratio, the smaller the impact of the estimation errors on portfolio performance is.

To better understand the connection of the Sharpe ratio with the length of the sample and the number of assets, I plot the expected SSR, measured by $\bar{\zeta}_{2}^{2}$, vs. T (Panels A and B) and $N$ (Panels $\mathrm{C}$ and $\mathrm{D}$ ) in Figure 1. In panels $\mathrm{A}$ and $\mathrm{C}$, the moments of the returns are calibrated in the 10 Ind set while, in the remaining panels, they are calibrated in the $25 \mathrm{SBM}$ set. I also plot the maximum SSR offered by the ex-ante tangency portfolio as well as the SSR for the equally-weighted portfolio $(1 / N)$. I consider the latter portfolio, motivated by the recent debate in the literature about the comparative performance between $1 / N$ and efficient portfolios. On the one hand, DeMiguel et al. (2009) find that the tangency portfolio heavily underperforms $1 / N$ out-of-sample in several cases. On the other hand, Kritzman et al. (2010) show that this issue can be resolved by using long datasets for estimating the moments of asset returns. The comparisons performed here can provide insights to this debate by identifying cases where one portfolio outperforms the other.

The graph confirms the positive (negative) relationship between the expected SSR of the tangency portfolio and $T(N)$. I also observe that, for 10 assets and $\theta^{2}=0.037$, at least 256 observations are required for the estimated tangency portfolio to outperform $1 / N$. Alternatively, for 20 years of monthly observations $(T=240)$ and $\theta^{2}=0.037$, the estimated tangency portfolio offers a higher SSR, only when the assets are less than 10 . When $\theta^{2}=$ 0.204 , however, the tangency portfolio is superior to $1 / N$ in all cases considered. This result confirms that the impact of estimation errors in the out-of-sample Sharpe ratio is smaller for large values of the maximum Sharpe ratio. The latter acts a moderating factor in the comparative performance between the tangency portfolio and $1 / N$. 
Figure 1: Sensitivity of the expected squared Sharpe ratio to the sample length and the number of assets
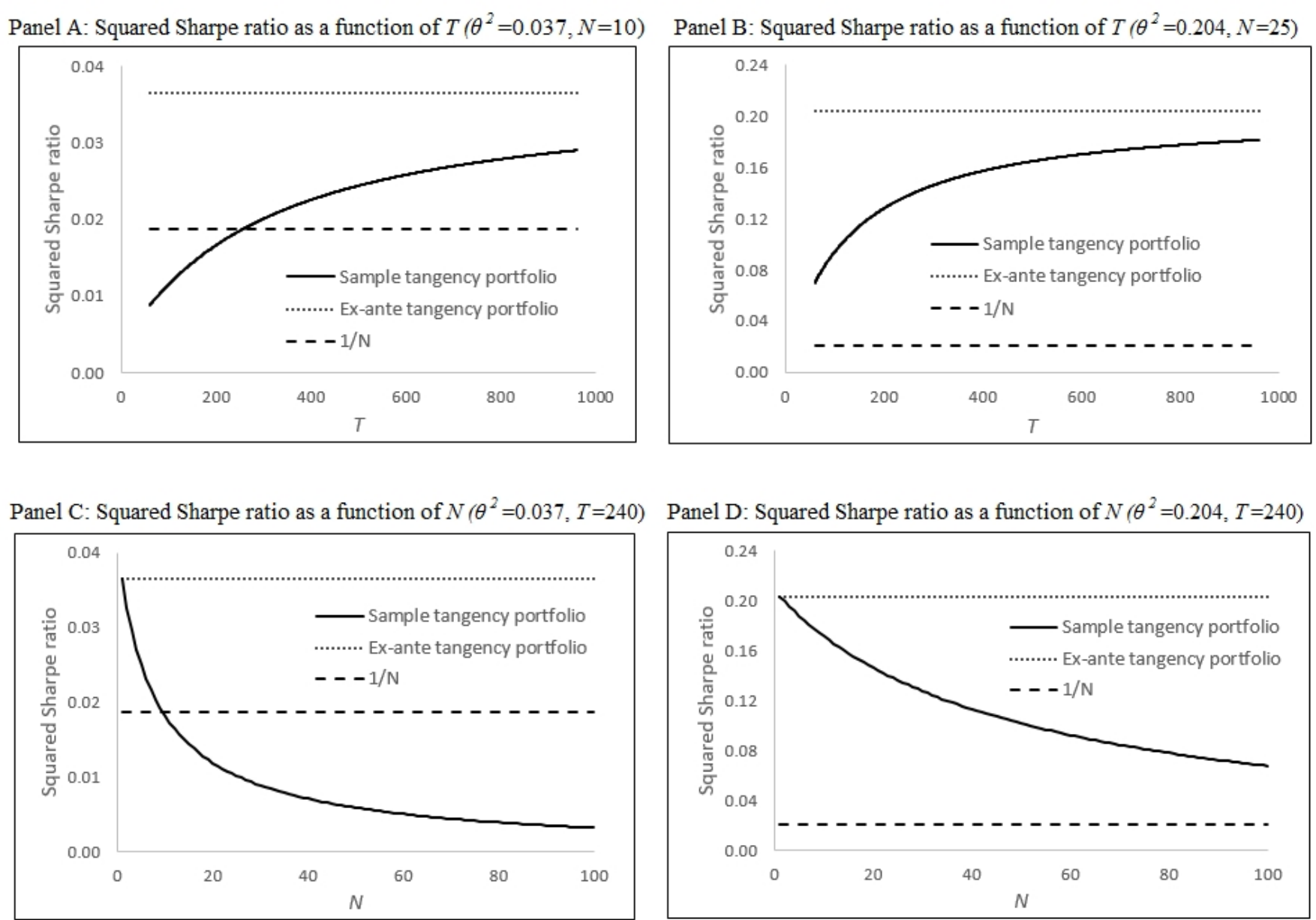

Note: This figure depicts the expected squared Sharpe ratio (SSR) of the estimated tangency portfolio vs. the sample size $T$ (Panels A and B) and the number of assets $N$ (Panels C and D). The expected SSR is computed using the formula provided in section $3\left(\bar{\zeta}_{2}^{2}\right)$. The SSRs of the ex-ante tangency portfolio $\left(\theta^{2}\right)$ and $1 / N$ are also presented. In Panels A and $\mathrm{C}(\mathrm{B}$ and $\mathrm{D})$, the moments of asset returns are calibrated in a set of 10 industry portfolios (25 portfolios formed on size and book-to-market). 


\section{Estimating the squared Sharpe ratio}

The analysis in this work enables the portfolio manager to estimate the expected SSR of estimated efficient portfolios and assess their suitability, given the investment setting. In particular, the manager can estimate $\theta^{2}$ based on the available data, and, in turn, estimate the expected out-of-sample SSR of the tangency portfolio using the expressions in Proposition 1. As an example of this approach, I consider the following estimator of $\theta^{2}$ :

$$
\tilde{\theta}^{2}=\max \left\{\hat{\theta}^{2}-\frac{N}{T}, \frac{2}{N+2} \hat{\theta}^{2}\right\}
$$

where $\hat{\theta}^{2}=\hat{\mu}^{\prime} \Sigma^{-1} \hat{\mu} .^{7}$ This estimator stems from the work of Kubokawa et al. (1993) and is based on the fact that $T \hat{\theta}^{2}$ follows a noncentral chi-squared distribution with $N$ degrees of freedom and noncentrality parameter $T \theta^{2}$. I then estimate the expected out-of-sample SSR $\left(\bar{\zeta}_{2}^{2}\right)$ and the corresponding expected loss in the $\operatorname{SSR}\left(\bar{\rho}_{2}\right)$ by respectively substituting $\tilde{\theta}^{2}$ for $\theta^{2}$ in (8) and (11). I denote the resulting estimators with $\hat{\zeta}_{2}^{2}$ and $\hat{\rho}_{2}$, respectively.

I study the performance of the derived estimators using the same datasets and considering the same scenarios as in Table 1. In Table 2, I present the mean and the standard deviation (in parenthesis) of the estimators, computed using again 100,000 samples of simulated returns. I also report the true values of $\bar{\zeta}_{2}^{2}$ and $\bar{\rho}_{2}$. I draw three conclusions from these results. First, the performance of the estimator of the expected SSR is significantly better for the dataset of 25 assets than for the set of 10 assets. For example, in the larger dataset, the bias of $\hat{\zeta}_{2}^{2}$ is less than $7 \%$ compared to about $43 \%$ in the smaller dataset. This finding indicates that the accuracy of $\hat{\zeta}_{2}^{2}$ increases with the true value of $\theta^{2} .8$ Second, the estimator of the expected loss performs significantly better than the estimator of the expected SSR, leading to smaller bias and standard deviation in most considered cases. Finally, the bias and the variance of the estimators decrease with the sample length, as one would expect.

\footnotetext{
${ }^{7}$ Alternatively, Kan and Zhou (2007) explore the estimation of $\theta^{2}$ when both $\mu$ and $\Sigma$ are unknown.

${ }^{8}$ This finding can be further confirmed through a simulation experiment. The results of this experiment are available upon request.
} 
Table 2: Statistics for the estimators of out-of-sample performance metrics

\begin{tabular}{cccccccc}
\hline & \multicolumn{4}{c}{ Expected SSR } & \multicolumn{3}{c}{ Expected loss in the SSR } \\
$N$ & $T$ & $\bar{\zeta}_{2}^{2}$ & $E\left(\hat{\zeta}_{2}^{2}\right)$ & (St. dev.) & $\bar{\rho}_{2}$ & $E\left(\hat{\rho}_{2}\right)$ & (St. dev.) \\
\hline \multirow{4}{*}{10} & 60 & 0.0088 & 0.0274 & $(0.0420)$ & 0.0278 & 0.0368 & $(0.0245)$ \\
& 120 & 0.0127 & 0.0222 & $(0.0291)$ & 0.0238 & 0.0233 & $(0.0140)$ \\
& 240 & 0.0182 & 0.0220 & $(0.0220)$ & 0.0184 & 0.0163 & $(0.0074)$ \\
& 480 & 0.0241 & 0.0250 & $(0.0170)$ & 0.0125 & 0.0115 & $(0.0029)$ \\
& & & & & & & \\
& 60 & 0.0700 & 0.0899 & $(0.0927)$ & 0.1337 & 0.1223 & $(0.0641)$ \\
25 & 120 & 0.1029 & 0.1100 & $(0.0750)$ & 0.1009 & 0.0945 & $(0.0278)$ \\
& 240 & 0.1364 & 0.1384 & $(0.0576)$ & 0.0674 & 0.0657 & $(0.0080)$ \\
& 480 & 0.1634 & 0.1637 & $(0.0420)$ & 0.0403 & 0.0400 & $(0.0018)$ \\
\hline
\end{tabular}

Note: This table presents the mean and standard deviation (in parenthesis) of the estimators of the expected squared Sharpe ratio (SSR) and the expected loss in the SSR from estimation errors for the estimated tangency portfolio, both derived as described in section 5. I consider two values for the number of assets $N$ and four for the sample length $T$. The underlying assumption is that asset returns are i.i.d. over time following a normal distribution with parameters calibrated in a set of 10 industry portfolios $(N=10)$ and a set of 25 portfolios formed on size and book-to-market $(N=25)$. The true value of the expected SSR $\left(\bar{\zeta}_{2}^{2}\right)$ and the expected loss $\left(\bar{\rho}_{2}\right)$, computed using the second-order approximation in Proposition 1 , are also reported.

\section{Conclusion}

Portfolio strategies that achieve superior Sharpe ratios are highly sought after in investment practice. While mean-variance analysis offers portfolios that maximize the Sharpe ratio, practical applications of efficient portfolios are problematic. This is because estimated efficient portfolios are subject to errors that lead to inferior portfolio performance. To study how estimation errors affect the Sharpe ratio, this article develops a set of simple and intuitive approximations of the expected squared Sharpe ratio of the estimated tangency portfolio. The expected squared Sharpe ratio increases with the number of assets and the maximum possible Sharpe ratio while it decreases with the length of the data in hand. My analysis enables the portfolio manager to estimate the average squared Sharpe ratio of estimated efficient portfolios. Then, if the manager expects a low squared Sharpe ratio, alternative portfolio strategies could be adopted.

This paper sets the ground for further research in three promising directions. First, fu- 
ture work could address a limitation of this work, i.e., the assumption of a known covariance matrix. Accounting for uncertainty about the covariance matrix is important in cases where the ratio of number of assets to the sample length is small. This is because in such cases estimation errors in the covariance matrix can have a large impact on portfolio performance, as Kan and Zhou (2007) show. Second, future work could develop estimators of the expected squared Sharpe ratio for other types of sample-based portfolio strategies, building on the ideas in this paper. Such estimators would enable investors to choose among different strategies on the basis of their expected squared Sharpe ratio. Third, in the same spirit as Kan and Zhou (2007), future research could use the methods in this study to construct estimators of efficient portfolios that offer higher out-of-sample Sharpe ratios.

\section{A Appendix: Proof of Proposition 1}

I set $M=\mu \mu^{\prime}$. Then, the SSR for the tangency portfolio can be written as

$$
\hat{\zeta}^{2}=\frac{\tilde{w}^{\prime} M \tilde{w}}{\tilde{w}^{\prime} \Sigma \tilde{w}}
$$

where $\tilde{w}=\Sigma^{-1} \hat{\mu}$. Since $\hat{\mu} \sim N(\mu, \Sigma / T), \tilde{w} \sim N\left(\Sigma^{-1} \mu, \Sigma^{-1} / T\right)$. Therefore, $\hat{\zeta}^{2}$ is a ratio of quadratic forms in normal variables. Let $X=\tilde{w}^{\prime} M \tilde{w}$ and $Y=\tilde{w}^{\prime} \Sigma \tilde{w}$. I can approximate $E=\frac{X}{Y}$ using Taylor series expansions (e.g, see Stuart and Ord, 1994, p. 351):

$$
\begin{gathered}
E\left(\frac{X}{Y}\right) \approx \frac{E(X)}{E(Y)} \text { (first-order) } \\
E\left(\frac{X}{Y}\right) \approx \frac{E(X)}{E(Y)}\left(1-\frac{\operatorname{Cov}(X, Y)}{E(X) E(Y)}+\frac{\operatorname{Var}(Y)}{E(Y)^{2}}\right) \text { (second-order). }
\end{gathered}
$$


The moments of $X$ and $Y$ are (e.g., see Paolella, 2003)

$$
\begin{gathered}
E(X)=\frac{\operatorname{tr}\left(M \Sigma^{-1}\right)}{T}+E(\tilde{w})^{\prime} M E(\tilde{w})=\frac{\mu^{\prime} \Sigma^{-1} \mu}{T}+\left(\mu^{\prime} \Sigma^{-1} \mu\right)^{2}=\theta^{2} \frac{1+T \theta^{2}}{T} \\
E(Y)=\frac{N}{T}+E(\tilde{w})^{\prime} \Sigma E(\tilde{w})=\frac{N}{T}+\mu^{\prime} \Sigma^{-1} \mu=\frac{N+T \theta^{2}}{T} \\
\operatorname{Var}(Y)=2 \frac{N}{T^{2}}+4 \frac{E(\tilde{w})^{\prime} \Sigma E(\tilde{w})}{T}=2 \frac{N}{T^{2}}+4 \frac{\mu^{\prime} \Sigma^{-1} \mu}{T}=2 \frac{N+2 T \theta^{2}}{T^{2}} \\
\operatorname{Cov}(X, Y)=2 \frac{\operatorname{tr}\left(M \Sigma^{-1}\right)}{T^{2}}+4 \frac{E(\tilde{w})^{\prime} M E(\tilde{w})}{T}=2 \theta^{2} \frac{1+2 T \theta^{2}}{T^{2}}
\end{gathered}
$$

given that $\operatorname{tr}\left(M \Sigma^{-1}\right)=\theta^{2}$ and $\left(\Sigma^{-1} \mu\right)^{\prime} M\left(\Sigma^{-1} \mu\right)=\theta^{4}$. Substituting (A.4)-(A.7) to (A.2)(A.3) gives (7) and (8).

\section{References}

Amenc, N., L. Martellini, and M. Vaissié. "Benefits and risks of alternative investment strategies." Journal of Asset Management, 4 (2003), pp. 96-118.

Chopra, V. K., and W. T. Ziemba. "The effect of errors in means, variances, and covariances on optimal portfolio choice," Journal of Portfolio Management, 19 (1993), pp. 6-11.

DeMiguel, V., L. Garlappi, and R. Uppal. "Optimal versus naive diversification: How inefficient is the $1 / N$ portfolio strategy?" Review of Financial Studies, 22 (2009), pp. 1915-1953.

Grinold, R. C., and R. N. Kahn. Active portfolio management, 2nd edition, NY: McGrawHill, 1999.

Kan, R., and G. Zhou. "Optimal portfolio choice with parameter uncertainty." Journal of Financial and Quantitative Analysis, 42 (2007), pp. 621-656.

Kritzman, M., S. Page, and D. Turkington. "In defense of optimization: The fallacy of 1/N." Financial Analysts Journal, 66 (2010), pp. 31-39. 
Kubokawa, T., C . P., Robert, and A. K., Saleh. "Estimation of noncentrality parameters." Canadian Journal of Statistics, 21 (1993), pp. 45-57.

Markowitz, H. "Portfolio selection." Journal of Finance, 7 (1952), pp. 77-91.

Merton, Robert C. "On estimating the expected return on the market: An exploratory investigation." Journal of Financial Economics, 8 (1980), pp. 323-361.

Meucci, A. Risk and asset allocation, NY: Springer-Verlag, 2005.

Michaud, R. O. "The Markowitz optimization enigma: Is optimized optimal?" Financial Analysts Journal, 45 (1989), pp. 31-42.

Miller, R. E., and A. K., Gehr. "Sample size bias and Sharpe's performance measure: A note." Journal of Financial and Quantitative Analysis, 13 (1978), pp. 943-946.

Lo, A. W. "The statistics of Sharpe ratios." Financial Analysts Journal, 58 (2002), pp. 36-52.

Paolella, M. S. "Computing moments of ratios of quadratic forms in normal variables." Computational Statistics \& Data Analysis, 42 (2003), 313-331.

Sharpe, W. F. "Mutual fund performance." Journal of Business, 39 (1966), pp. 119-138.

Sharpe, W. F. "The Sharpe ratio." Journal of Portfolio Management, 21 (1994), pp. 49-58.

Stuart, A., and J.K., Ord. Kendall's Advanced Theory of Statistics, Volume 1, Distribution Theory, 6th edition, Halsted Press, John Wiley and Sons, New York, 1994.

Treynor, J. L., and F. Black. "How to use security analysis to improve portfolio selection." Journal of Business, 46 (1973), pp. 66-86.

Tu, J., and G. Zhou. "Markowitz meets Talmud: A combination of sophisticated and naive diversification strategies.” Journal of Financial Economics, 99 (2011), pp. 204-215. 